\title{
Time-Resolved XPS Analysis of the $\mathrm{SiO}_{2} / \mathrm{Si}$ System in the Millisecond Range
}

\author{
U. Korcan Demirok, Gulay Ertas, and Sefik Suzer* \\ Bilkent University, Chemistry Department, 06800 Ankara, Turkey \\ Received: February 3, 2004; In Final Form: March 5, 2004
}

\begin{abstract}
By applying voltage pulses to the sample rod while recording the spectrum, we show, for the first time, that it is possible to obtain a time-resolved XPS spectrum in the millisecond range. The Si $2 p$ spectrum of a silicon sample containing a ca. 400-nm oxide layer displays a time-dependent charging shift of ca. $1.7 \mathrm{eV}$ with respect to the $\mathrm{Au} 4 \mathrm{f}$ peaks of a gold metal strip in contact with the sample. When gold is deposited as $\mathrm{C}_{12}$-thiol-capped nanoclusters onto the same sample, this time the $\mathrm{Au} 4 \mathrm{f}$ peaks also display time-dependent charging behavior that is slightly different from that of the Si $2 p$ peak. This charging/discharging is related to emptying/filling of the hole traps in the oxide layer by the stray electrons within the vacuum system guided by the external voltage pulses applied to the sample rod, which can be used to extract important parameter(s) related to the dielectric properties of surface structures.
\end{abstract}

Charging/discharging is one of the fundamental processes dictating both the thermodynamics and the kinetics of the various physicochemical changes taking place on surfaces. ${ }^{1}$ In addition to the standard electrical measurements, STM, AFM, and Kelvin probe techniques are widely used to elucidate the structural changes accompanying these processes. ${ }^{2-6}$ When chemical information is also needed, XPS is usually the preferred spectroscopic technique because all elements, except for hydrogen, and their oxidation state(s) can easily be identified. ${ }^{7}$ One of the disadvantages of the XPS technique is that additional positive charges are naturally introduced as a consequence of the photoemission process. These charges do not interfere with measurements when the surfaces are electrically conducting but can cause significant binding-energy shifts for poorly conducting samples. ${ }^{8-11}$ Such charges are usually compensated by flooding the sample with low-energy electrons (or sometimes ions) and trying to bring the surfaces to a steady state in terms of electrons going in and out, but the complete elimination of charging is only an ideal. Surfaces can also be negatively charged if more electrons are introduced. This modification, dubbed controlled surface charging (CSC), has been successfully applied to depth profiling in the $1-10 \mathrm{~nm}$ range and/or to the lateral differentiation of mesoscopic layers. ${ }^{12-16}$ However, prolonged exposure of the surfaces to intense low-energy electrons can cause physical and chemical damage. ${ }^{9}$ It was even reported that the surface potential that developed from the added charging caused deintercalation in layered compounds. ${ }^{17} \mathrm{Lau}$ and co-workers have also utilized the CSC to extract structural and electrical properties of ultrathin dielectrics on semiconductors. ${ }^{18-22}$ In a complementary study, we have recently reported that the positive charging developed on the $\mathrm{SiO}_{2} / \mathrm{Si}$ system can also be controlled simply by the application of an external bias to the sample rod to affect the measured binding-energy difference between the $\mathrm{Si}^{4+}$ overlayer and the $\mathrm{Si}^{0}$ underlayer; the positive bias that was applied attracted stray electrons within the vacuum system, originating mostly from the X-ray tube, and caused partial neutralization of the positive charge developed, resulting in a smaller binding-energy difference. Negative bias acts in the opposite way. ${ }^{23}$ By controlling the differential charging with

\footnotetext{
* Corresponding author. E-mail: suzer@ fen.bilkent.edu.tr.
}

external biasing, we were also able to (i) separate otherwise overlapping XPS peaks of the same atom belonging to different chemical entities and (ii) derive information related to the dielectric properties of the layers and the proximity of the atoms within composite multilayers. ${ }^{24,25}$

Instead of applying the bias in the dc mode, we can pulse it for time-resolved measurements, which is the main subject of the present contribution. Except for the very fast $\left(10^{-9}-10^{-15}\right.$ s) laser-induced (optical) processes, ${ }^{26-32}$ conventional XPS data is recorded in the $10^{1}-10^{4} \mathrm{~s}$ range because of the very low cross section of the photoemission process even with the use of strong synchrotron radiation sources. ${ }^{33}$ There are also various processes taking place in the millisecond range that are of importance, and the time-resolved mode can easily be implemented using available PC controller cards. The present work is the first dealing with time-resolved XPS measurements on solid surfaces in the millisecond range.

Figure 1 depicts schematically our pulse sequences together with the XPS spectra of the sample (in electrical contact with a gold metal strip for referencing) recorded under +10 and -10 $\mathrm{V}$ dc bias separately. We have chosen a silicon sample containing a thick (ca. $400 \mathrm{~nm}$ ) oxide layer to amplify the charging for a clearer demonstration of the technique. Hence, only the Si 2 p peak of the oxide layer and the Au $4 \mathrm{f}$ spinorbit doublet are observable (i.e., the Si substrate is too deep to be probed by XPS). As also given in the figure, the measured binding-energy differences between the $\mathrm{Au} 4 \mathrm{f}$ and the Si $2 \mathrm{p}$ are 19.4 and $21.1 \mathrm{eV}$ under +10 and $-10 \mathrm{~V}$ bias, respectively, due to the different potential developed (as a result of different charging) in each case.

The pulses are applied to the sample rod externally using a digital-to-analog converter (DAC) card, and the photoelectrons are amplified and counted for a given period ( $5 \mathrm{~ms}$ in this case) and stored. The kinetic energy axis of the spectrometer is controlled from the second DAC port of the same card. A computer program controls the pulses and stores the data. Each measurement starts by setting the kinetic energy to the first point and applying $+10 \mathrm{~V}$ to the sample rod. We then apply four repetitive steps: (1) wait for $2.5 \mathrm{~s}$ for equilibration, (2) suddenly change the applied voltage to $-10 \mathrm{~V}$, and (3) start recording 


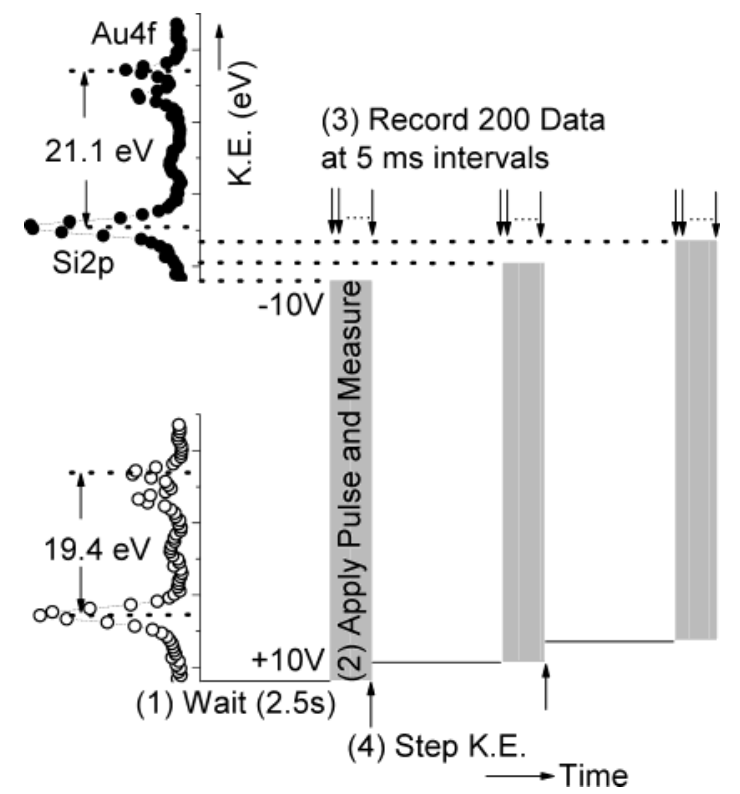

Figure 1. Pulse sequences used for recording XPS spectra in 5-ms time-resolved mode. XPS spectra of the Si $2 p-A u 4 f$ region recorded under -10 and $+10 \mathrm{~V}$ dc bias separately are given on the side for illustration purposes. The actual time-resolved spectra are recorded only when $-10 \mathrm{~V}$ is applied to the rod.

the counts for $5 \mathrm{~ms}$ in separate columns for 200 data points. After the completion of data recording for $1 \mathrm{~s}$, the voltage is returned to $+10 \mathrm{~V}$, followed by (4) incrementing the energy to start the cycle for the next kinetic energy. One spectrum scan is completed only after repeating these cycles for the entire XPS region ( $\mathrm{Si} 2 \mathrm{p}$ and $\mathrm{Au} 4 \mathrm{f}$ in this case). Because only ca. 10-50 counts are collected in the peak, signal averaging is necessary. Typically, 50-250 scans are recorded and added, which lasts $3-15 \mathrm{~h}$. The resultant is a collection of 200 spectra recorded with 5-ms time resolution (and averaged over 100 scans in this case) as given in Figure 2, adding a new dimension (time) to the XPS analysis. A multitude of surface chemical/structural parameters can now be derived from the time correlation of different atoms, which is similar to powerful 2D NMR, Raman, or IR spectroscopic techniques. In principle, the time resolution is limited only by the rise time of the applied pulses, which is on the order of $0.01 \mathrm{~ms}$ for the DAC card we used in this work. However, in our case the 5-ms resolution limit was dictated by a combination of the counting statistics and the software we used.

As shown in Figure 2a, the Si 2p peak undergoes completely reversible (because this is a collection of 100 different spectra) time-dependent charging whereas the $\mathrm{Au} 4 \mathrm{f}$ of the gold metal strip does not. In addition, no measurable broadening or any other inhomogeneous response could be detected on the $\mathrm{Au}$ 4f peaks as a result of recording in the time-resolved mode. In Figure 2b, we also display another 200 time-resolved spectra of the same $\mathrm{SiO}_{2} / \mathrm{Si}$ sample onto which $\mathrm{C}_{12}$-thiol-capped gold nanoclusters are deposited from an aqueous medium. ${ }^{34}$ In this case, the Au $4 \mathrm{f}$ peaks of the gold clusters also display timedependent charging that is slightly different from that of the $\mathrm{Si}$ $2 p$ of the oxide underlayer as shown at the top of Figure $2 c$. A reversal of the polarity of the applied pulses leads to discharging curves with similar time behavior.

The initial photoemission and subsequent filling of the photohole are extremely fast (shorter than $10^{-12} \mathrm{~s}$ ) processes. Therefore, what we observe in the millisecond range is mostly related to the trapping and detrapping of the holes in the valence band of the oxide layer by the low-energy stray electrons. ${ }^{35}$

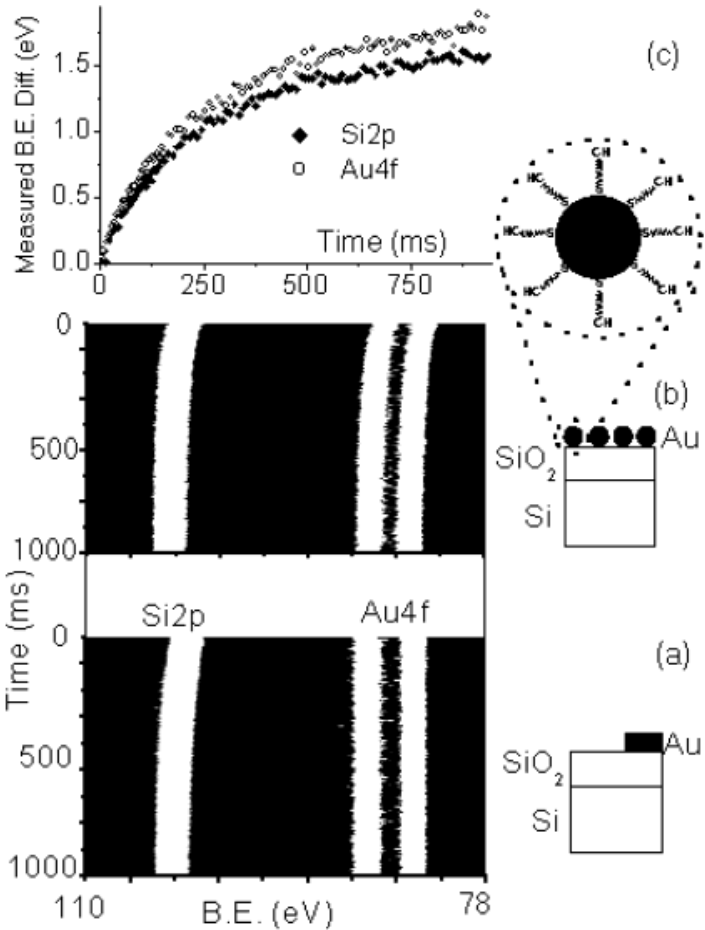

Figure 2. Time-resolved XPS spectra (200) of the Si $2 \mathrm{p}-\mathrm{Au} 4 \mathrm{f}$ region of (a) a silicon sample containing a ca. 400-nm oxide layer and a gold metal strip connected to it and (b) the same sample onto which $\mathrm{C}_{12-}$ thiol-capped gold nanoclusters are deposited. Resultant binding-energy shifts, which are obtained by curve fitting of the individual time-resolved spectra of $b$, are shown for the Si $2 p$ and $\mathrm{Au} 4 \mathrm{f}$ peaks in Figure 2c.

Because of the detrimental effects of X-rays on the performance of metal oxide-semiconductor (MOS) systems, such processes have long been studied by measuring the time-dependent leakage current $^{36-38}$ and recently by scanning capacitance microscopy (SCM). The time constant we derive from our measurements, in the 100-200 ms range, is in agreement with the lower end of the values reported $\left(0.1-10^{3} \mathrm{~s}\right)$ using the scanning capacitance technique. ${ }^{39}$ The main purpose of the present contribution is the introduction of the technique. Hence, chemical/structural parameters derivable using this technique will be the subject of our future investigation(s).

In conclusion, by implementing voltage pulses applied externally to the sample rod, we were able to record XPS spectra in time-resolved mode (in the millisecond range) to investigate the charging/discharging of the oxide layer on silicon and the gold clusters on top of them. In addition to the conventional chemical information derived from XPS analysis, time-dependent measurements can now be used to extract important parameters related to dielectric properties (capacitance, relaxation constant, etc.) of surface structures.

Acknowledgment. We thank Professor Ziya Ider of Bilkent University for valuable discussions and the TUBA (Turkish Academy of Sciences) for partial financial support.

\section{References and Notes}

(1) Adamson, A. W. Physical Chemistry of Surfaces, 5th ed.; Wiley: New York, 1990.

(2) Ingram, R. S.; Hostetler, M. J.; Pietron, J. J.; Murray, R. W. Schaaff, T. G.; Khoury, J. T.; Whetten, R. L.; Bigioni, T. P.; Guthrie, D. K.; First, P. N. J. Am. Chem. Soc. 1997, 119, 9279.

(3) Chen, S.; Ingram, R. S.; Hostetler, M. J.; Pietron, J. J.; Murray, R. W.; Schaaff, T. G.; Khoury, J. T., Alvarez, M. M.; Whetten, R. L. Nature 1998, 280, 2098.

(4) Ohgi, T.; Fujita, D. Phys. Rev. B 2002, 66, 115410. 
(5) Mao, D.; Kahn, A.; Marsi, M.; Margaritondo, G. Phys. Rev. B 1990 , $42,3228$.

(6) Rampi, M. A.; Schueller, O. J. A.; Whitesides, G. M. Appl. Phys. Lett. 1998, 72, 1781.

(7) Briggs, D.; Seah, M. P. Practical Surface Analysis, 2nd ed.;

Wiley: Chichester, England, 1999; Vol. 1.

(8) Barr, T. L. Crit. Rev. Anal. Chem. 1991, 22, 229.

(9) Cros, A. J. Electron Spectrosc. Relat. Phenom. 1992, 59, 1.

(10) Cazaux, J. J. Electron Spectrosc. Relat. Phenom. 1999, 105, 155. Cazaux, J. J. Electron Spectrosc. Relat. Phenom. 2000, 113, 15.

(11) Larson, P. E.; Kelly, M. A. J. Vac. Sci. Technol., A 1998, 16, 3483.

(12) Shabtai, K.; Rubinstein, I.; Cohen, S. R.; Cohen, H. J. Am. Chem. Soc. 2000, 122, 4959 .

(13) Doron-Mor, I.; Hatzor, A.; Vaskevich, A.; Van der Boom-Moav, T.; Shanzer, A.; Rubinstein, I.; Cohen, H. Nature 2000, 406, 382.

(14) Buller, R.; Cohen, H.; Minkin, E.; Popovitz-Biro, R.; Lifshitz, E.; Lahav, M. Adv. Funct. Mater. 2002, 12, 713.

(15) Samokhvalov, A.; Gurney, R. W.; Lahav, M.; Cohen, S.; Cohen,

H.; Naaman, R. J. Phys. Chem. B 2003, 107, 4245.

(16) Shabtai, K.; Cohen, S. R.; Cohen, H.; Rubinstein, I. J. Phys. Chem. B 2003, 107, 5540.

(17) Feldman, Y.; Zak, A.; Tenne, R.; Cohen, H. J. Vac. Sci. Technol., A 2003, 21, 1752 .

(18) Lau, W. M. J. Appl. Phys. 1989, 65, 2047.

(19) Lau, W. M. Appl. Phys. Lett. 1989, 54, 338

(20) Lau, W. M.; Jin, S.; Wu, X. W.; Ingrey, S. J. Vac. Sci. Technol., $B$ 1990, 8, 848 .

(21) Chan, R. W. M.; Kwok, R. W. M.; Lau, W. M. J. Appl. Phys. 1996, 79, 3635 .

(22) Chan, R. W. M.; Kwok, R. W. M.; Lau, W. M.; Yan, H.; Wong, S. P. J. Vac. Sci. Technol., A 1997, 15, 2787.
(23) Ulgut, B.; Suzer, S. J. Phys. Chem. B 2003, 107, 2939.

(24) Karadas, F.; Ertas, G.; Suzer, S. J. Phys. Chem. B 2004, 108, 1515.

(25) Suzer, S. Anal. Chem. 2003, 75, 7026.

(26) Alonso, M.; Cimino, R.; Horn, K. Phys. Rev. Lett. 1990, 64, 1947.

(27) Baumler, M.; Haight, R. Phys. Rev. Lett. 1991, 67, 1153.

(28) Rattenberger, A.; Leiderer, P.; Probst, M.; Haight, R. Phys. Rev. B 1997, 56, 12092.

(29) Mihaychuck, J. G.; Shamir, N.; van Driel, H. M. Phys. Rev. B 1999, 59, 2164.

(30) Marsi, M.; Belkhou, R.; Grupp, C.; Panaccionne, G.; Taleb-Ibrahimi, A. Nahon, L.; Garzella, D.; Nutarelli, D.; Renault, E.; Roux, R.; Couprie, M. E.; Billardon, M. Phys. Rev. B 2000, 61, R5070.

(31) Nugent-Glandorf, L.; Scheer, M.; Samuels, D. A.; Mulhisen, A. M., Grant, E. R.; Yang, X.; Bierbaum, V. M.; Leone, S. R. Phys. Rev. Lett. 2001, 87, 193002.

(32) Bauer, M.; Lei, C.; Read, K.; Tobey, R.; Gland, J.; Murnane, M. M.; Kapteyn, H. C. Phys. Rev. Lett. 2001, 87, 025501.

(33) Hirose, K.; Sakano, K.; Takahashi, K.; Hattori, T. Surf. Sci. 2002, 507-510, 906.

(34) Brust, M.; Walker, M.; Bethell, D.; Schiffrin, D. J.; Whyman, R. J. Chem. Soc., Chem. Commun. 1994, 801.

(35) Iwata, S.; Ishizaka, A. J. Appl. Phys. 1996, 79, 6653

(36) Curtis, O. L., Jr.; Srour, J. R. J. Appl. Phys. 1977, 48, 3819.

(37) Hughes, R. C. Phys. Rev. B 1977, 15, 2012.

(38) Wang, T.; Cheng, T. E.; Chiang, L. P.; Wang, C. H.; Zons, N. K.; Huang, C. IEEE Trans. Electron Devices 1998, 45, 1511.

(39) Kang, C. J.; Buh, G. H.; Lee, S.; Kim, C. K.; Mang, K. M.; Im, C.; Kuk, Y. Appl. Phys. Lett. 1999, 74, 1815. 